ISSN 2447-9071

doi https://doi.org/10.36414/rbmc.v6i15.35

Contato para correspondência: Leonardo Luiz Borges

E-mail:

leonardo.cbb@pucgoias.edu.br

Conflito de interesse: Não

Financiamento: Recursos próprios

Recebido: 02/04/2020

Aprovado: 29/06/2020

\section{Importância da atenção farmacêutica para a quimioterapia antitumoral}

\section{Importance of pharmaceutical care for antitumoral chemotherapy}

\author{
Erica Assis Alves ${ }^{1}$, Gabriel Guimarães Tavares ${ }^{1}$, Leonardo Luiz Borges ${ }^{1,2}$ \\ 'Pontifícia Universidade Católica de Goiás - PUC Goiás \\ 2 Universidade Estadual de Goiás - UEG
}

\section{Resumo}

Esta revisão teve como objetivo destacar a importância do profissional farmacêutico nos cuidados clínicos de um paciente oncológico, visando a sua melhoria de qualidade de vida e maior efetividade da farmacoterapia no tratamento do câncer. E descrever resumidamente sobre as principais drogas utilizadas no tratamento dos diversos tipos de câncer. Foi realizada uma revisão bibliográfica nas principais plataformas de bases, selecionando publicações de 2009 a 2019. Dos resultados obtidos foram selecionados 32 artigos, duas teses de doutorado, 2 dissertações de mestrado e três trabalhos de conclusão de curso. Podemos concluir que o farmacêutico atuante da área de oncologia, deve ser um profissional comprometido em cuidar de paciente debilitados, fragilizados fisicamente e psicologicamente, também deve saber orientar tanto os pacientes quanto seus familiares, utilizando palavras sensatas e de conforto, portanto é um profissional indispensável na equipe multidisciplinar da oncologia.

Palavras-Chave: Atenção Farmacêutica, Câncer, Oncologia.

\begin{abstract}
This review aims to highlight the importance of the pharmaceutical professional in the medical care of an oncology patient, to apply his improvement in the quality of life and greater effectiveness of pharmacotherapy in the treatment of cancer. And briefly describe the main drugs used to treat various types of cancer. The review was performed on the main base platforms, selecting publications from 2009 to 2019. The results were selected from 32 articles, two doctoral theses, two master's dissertations and three course completion papers. If concluded that the pharmacist working in the area of oncology, must be a professional committed to caring for debilitated patients, physically and psychologically weakened, he must also know how to guide both patients and their families, using sensitive and comfort words, so he is a professional indispensable in the multidisciplinary oncology team.
\end{abstract}

Keywords: Pharmaceutical Care, Oncology, Cancer. 


\section{Introdução}

O câncer (neoplasia) pode ser definido como um conjunto de mais de 100 patologias, que é caracterizado pela disposição desordenada de células anormais (malignas) que invadem órgãos e tecidos adjacentes, causando a metástase ${ }^{1,2}$. Um tumor benigno por sua vez, é definido como uma massa de células localizadas no tecido ou órgão original. $O$ tratamento medicamentoso para o tumor pode gerar um problema relacionado com medicamento (PRM) que é definido como um evento ou circunstância que, ligado à farmacoterapia, pode interferir, real ou potencialmente, nos resultados esperados num determinado paciente ${ }^{3}$.

Os quimioterápicos antitumorais têm como objetivo destruir as células tumorais, mas devido a ação inespecífica desses quimioterápicos lesam tanto células saudáveis (principalmente células de crescimentos rápidos) quantos as tumorais, podendo causar os efeitos colaterais nos pacientes como náuseas, vômitos, mal-estar, perda de cabelo e susceptibilidade maior às infecções ${ }^{4}$.

A atenção farmacêutica (AF), com foco no paciente, surge como estratégia que almeja melhorar a qualidade do processo de utilização de medicamentos alcançando resultados satisfatórios. Atenção farmacêutica é definida como um conjunto de práticas farmacêuticas que visa a orientação ao paciente, com finalidade de aumentar a efetividade do tratamento medicamentoso, concomitante à detecção de Problemas Relacionados a Medicamentos (PRM) ${ }^{5}$. O objetivo da AF é garantir uma farmacoterapia racional, segura e custoefetiva. A AF envolve parâmetros de macrocomponentes como promoção e educação em saúde, orientação e atendimento farmacêutico, dispensação de medicamentos e acompanhamento farmacoterapêutico. A atenção farmacêutica também tem a função de registrar as atividades, mensurar e avaliar os resultados de PRM 6 .

O farmacêutico tem um papel importante no tratamento e acompanhamento farmacoterapêutico do paciente, prestando ao paciente uma atenção farmacêutica, garantido que a terapia medicamentosa do paciente está adequada, tornando o tratamento eficaz e melhorando a qualidade de vida do doente ${ }^{7}$.

Em uma equipe multidisciplinar, a presença do farmacêutico é indispensável, pois o mesmo além da função de elaborar manuais de normas e procedimentos farmacêuticos, ajuda a melhorar e diminuir os erros de medicações na prescrição dos antitumorais e ajuda também no monitoramento dos eventos adversos causados por medicamentos ${ }^{8}$.

O farmacêutico também atua na verificação de interações medicamentosas, já que geralmente pacientes oncológicos utilizam vários medicamentos para o tratamento da neoplasia.
A interação medicamentosa pode comprometer a eficácia do tratamento e pode diminuir a segurança do paciente, com isso pode causar altos custos para o hospital e até mesmo para o paciente, porque pode levar a vários dias de internação e com isso diminui a qualidade de vida do paciente ${ }^{9}$. O farmacêutico que atua na área da oncologia, além de participar da gestão da farmácia também possui a função de discutir juntamente com sua equipe multidisciplinar, sobre os procedimentos oncológicos mais aprimorados para cada paciente ${ }^{10}$.

Este artigo tem como objetivo destacar a importância do profissional farmacêutico nos cuidados clínicos de um paciente oncológico, visando a sua melhoria de qualidade de vida e maior efetividade da farmacoterapia no tratamento do câncer. E descrever resumidamente sobre as principais drogas utilizadas no tratamento dos diversos tipos de câncer.

\section{Métodos}

Foi realizada uma revisão bibliográfica por meio de pesquisa de artigos científicos e teses em bases de dados sobre a Atenção Farmacêutica na oncologia.

As bases de dados utilizadas foram: Scientific Electronic Library Online (SCIELO), Periódicos CAPES e Google Acadêmico. A pesquisa de publicações foi feita durante os meses de Janeiro e Fevereiro de 2019, e foram aceitas apenas publicações dos anos de 2009 a 2019. As palavras-chave empregadas foram "atenção farmacêutica", "oncologia" e "câncer".

Neste presente trabalho, primeiramente foram definidos os principais medicamentos usados no tratamento da neoplasia, de acordo com o Instituto Oncoguia e logo após os principais tipos de neoplasias que essas drogas tratam.

Os autores recorreram ao site Drugs.com para verificar as interações medicamentosas, alimentar e interações com doenças, explicando as principais consequências dessas interações e citaram pelo menos um exemplo de interações medicamentosas graves existentes dessas interações.

\section{Busca dos artigos científicos}

Os estudos levantados nesta revisão sistemática avaliaram a importância da atenção farmacêutica na quimioterapia antitumoral e identificou 32 artigos, três Trabalho de Conclusão de Curso (TCC), duas teses de doutorado e duas dissertações de mestrados distribuídos nas seguintes bases de dados: 29 no Google Acadêmico, 4 no Scielo, 3 no PubMed e 2 no LILACS. Como pode ser observado na figura 1. 


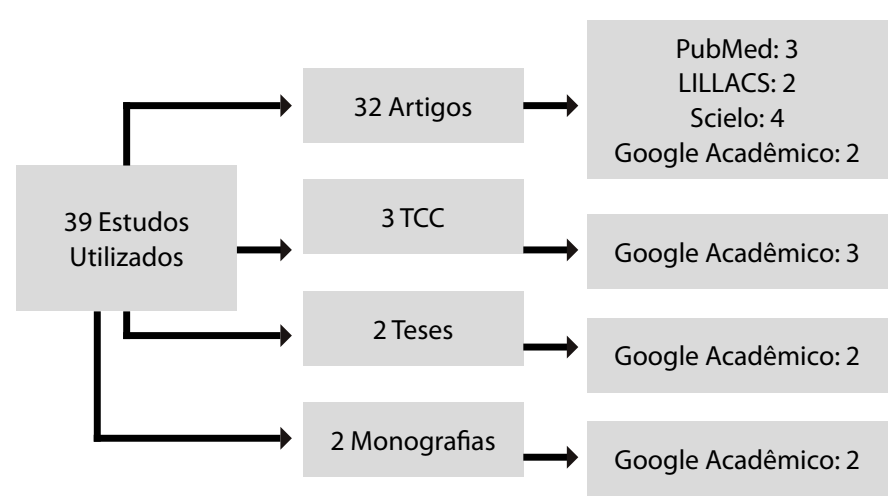

Figura 1. Esquema da Quantidade de Estudos Utilizados.

\section{Resultados e discussão}

\section{O câncer e seu impacto na saúde pública}

O câncer é um problema de saúde pública mundial, devido a sua alta incidência, o governo tem feitos altos investimentos para promover à saúde, na ajudar detecção precoce, na assistência à saúde, entre outros investimentos, para tentar reduzir a incidência dessa doença ${ }^{11}$.

De acordo com o Instituto Nacional de Câncer (INCA), estima-se mundialmente que em 2012 que houve 14,1 milhões de novos casos e 8,2 milhões de óbitos, com incidência de câncer de pulmão (1,8 milhão), seguido de câncer de mama (1,7 milhão). Nos homens os tipos de cânceres mais frequentes foram o de pulmão cerca de $16,7 \%$ e de próstata $15,0 \%$. Já nas mulheres os mais prevalentes foram mama $(25,2 \%) \mathrm{e}$ intestino $(9,2 \%)^{12}$.

Ainda segundo este instituto, no Brasil, os anos de 2018 e 2019 foram registrados 600 mil novos casos de câncer (para cada ano). Sendo a maior prevalência em homens o câncer de próstata $(68,2 \%)$ e nas mulheres o câncer de mama é o prevalente $(59,7 \%)^{12}$.

\section{O cuidado farmacêutico na quimioterapia} antineoplásica

O uso do AH se popularizou nos últimos anos por ser biocompatível e razoavelmente durável. O conhecimento detalhado da anatomia facial e o planejamento estético é de suma importância para devolver ao rosto um aspecto jovial, harmonioso e natural. Nenhum preenchedor é ideal para todas as áreas de aplicação na rotina clínica; cada preenchedor possui a profundidade cutânea que deve ser injetado ${ }^{23}$.

O nível de reticulação do AH e a profundidade da injeção dependem do local a ser injetado e o efeito desejado. Este efeito se estende desde o preenchimento de rugas superficiais até a remodelação de certas partes do rosto ${ }^{13}$.
A terapia oncológica vem se tornando altamente especializada e avançada, nos últimos anos, portanto para o paciente oncológico tenha um melhor atendimento e tratamento ele deve ser atendido e acompanhado por uma equipe composta por médicos, enfermeiros, farmacêuticos e outros profissionais da área da saúde ${ }^{13}$.

$\mathrm{O}$ avanço científico fez surgir novos medicamentos, favorecendo a maior necessidade de participação do farmacêutico no acompanhamento farmacoterapêutico dos pacientes em tratamento oncológico ${ }^{14}$.

O tratamento para o câncer compreende diferente formas: cirurgia, radioterapia, quimioterapia, imunoterapia, hormonioterapia e terapia alvo, esses três últimos o tratamento se dá por administração medicamentosa via oral, necessitando, portanto, da presença de um profissional especializado na orientação do uso desses produtos ${ }^{15}$. O uso dos medicamentos antineoplásicos orais vem se tornando uma ótima opção para o tratamento dos pacientes com câncer, pois possui algumas vantagens com relação ao uso de medicamentos intravenoso, é uma via de administração extremamente simples e rápida, econômica, não invasiva, os pacientes podem utilizar os medicamentos em seu próprio domicilio, melhorando a qualidade de vida do paciente, pois permite a continuidade de suas atividades e convívio com seus familiares, portanto aumenta a chance de adesão ao tratamento ${ }^{14,16}$. $O$ tratamento medicamentoso via oral também é indolor e possui menor efeito colateral se comparado com quimioterápicos administrados por outras vias ${ }^{15}$.

A ausência do seguimento farmacoterapêutico pode aumentar as taxas de mortalidade e/ou recidiva do câncer, portanto seguir corretamente o tratamento oncológico é importante para a cura da doença ${ }^{14}$.

A intervenção cirúrgica é uma experiência assustadora para o paciente e para seus familiares. Os pacientes podem apresentar tensão, ansiedade, medo (da anestesia, da mutilação e até mesmo da morte). E ainda segundo esses autores quanto maior o estresse e/ou a tensão antes da cirurgia, maior será as dificuldades enfrentar os limites e problemas que a cirurgia pode impor no cotidiano do paciente ${ }^{15}$.

Os fatores que podem estar relacionados a ansiedade do procedimento cirúrgico são: preocupações com as lesões que podem ocorrer, receio de dor no pós-operatório, incapacidade física e consequentemente dependência física, medo da anestesia e das complicações pós-cirúrgicas ${ }^{17}$.

A atenção farmacêutica baseia-se em educação em saúde, orientação, assistência, dispensação de fármacos, além do acompanhamento farmacoterapêutico ${ }^{18}$. 
Principais medicamentos prescritos para o tratamento do câncer de mama

\section{Capecitabina:}

A capecitabina é uma droga antineoplásica de ação citotóxica, indicada para o tratamento do câncer de mama (monoterapia ou em associação com outros antineoplásicos), câncer de colón e reto e câncer de estômago. É um pró-fármaco da fluropirimidina que é convertida enzimaticamente em 5-fluorouracil (5-FU) ${ }^{19}$. No nível celular, essa droga impede a formação de timidilato a partir do uracil, que é o precursor do trifosfato de timidina, essencial para o processo de síntese do DNA. Interfere também na síntese do RNA e das proteínas celulares ${ }^{20}$.

Essa droga apresenta importantes reações adversas como diarreia (frequentemente severa), síndrome mão-pé (caracterizada por adormecimento, formigamento, inchaço e vermelhidão nas palmas das mãos e nas solas dos pés ${ }^{20}$. Pode causar também náuseas, dor abdominal e mielosupressão (neutropenia prolongada) ${ }^{21}$.

Este medicamento possui um total de 285 interações medicamentosas, com 54 delas graves, como por exemplo varfarina e ácido fólico. Alimentos que possuem em sua composição ácido fólico podem aumentar os efeitos da capecitabina, podendo ter efeitos colaterais graves como anemia, sangramentos ${ }^{22}$.

O profissional farmacêutico deve alertar que pacientes que necessitam utilizar esses 2 medicamentos simultaneamente devem ser monitorados com maior atenção, com os parâmetros de coagulação aferidos e com o eventual ajuste de dose do anticoagulante. Os pacientes devem ser alertados a relatar qualquer sinal de hemorragia, incluindo dores, inchaço, cefaleias, aumento do fluxo menstrual, hemorragias nasais, presença de sangue na urina ou nas fezes. Além disso, o farmacêutico deve alertar que produtos com ácido fólico podem aumentar os efeitos da capecitabina ${ }^{22}$.

Existem 6 interações com doenças sendo elas, infecções (facilitando a disseminação de infecções devido a mielossupressão), doença arterial coronariana (desencadeando isquemia e angina), mielosupressão, disfunção renal (contraindicada para pacientes com disfunção renal grave), desidratação e disfunção hepática (em caso de disfunção hepática sua biodisponibilidade pode aumentar até $60 \%)^{22}$.

\section{Tamoxifeno:}

Essa droga é um agente antietrogênio utilizado no tratamento do câncer de mama, e mais recentemente, na quimioprevenção em mulheres com elevado risco desse tipo de câncer. Geralmente é prescrito após o tratamento cirúrgico ou quimioterápico convencional, como tratamento adjuvante sistêmica ${ }^{23}$. Atua bloqueando o sítio de ligação do estrogênio em vários tecidos, o que acaba por sua vez impedindo o estrogênio de realizar sua síntese de RNA e proteínas intracelulares, levando a consequência de não proliferação de metástases ${ }^{24}$.

Os principais efeitos colaterais relatados pelas pacientes foram: ondas de calor, sangramento vaginal, prurido vulvar e corrimento vaginal, erupção cutânea, intolerância gastrintestinal, inflamação do tumor, tontura e, ocasionalmente, retenção de fluidos e alopecia ${ }^{23}$. Pode causar também eventos vasculares trombóticos e risco de carcinoma do endométrio ${ }^{24}$.

O tamoxifeno possui 460 interações medicamentosa sendo 132 delas interações graves, como o escitalopram. 0 farmacêutico juntamente com a equipe médica deve analisar a possibilidade de utilização desses dois medicamentos simultaneamente, pois podem aumentar o risco de arritmias, devendo então monitorar os batimentos cardíacos do paciente. Esse profissional também deve informar aos pacientes a relatarem quaisquer sintomas que estiverem apresentando, e caso eles relatarem sintomas como tontura, desmaio, palpitações e falta de ar deve se comunicar a sua equipe multidisciplinar para verificarem os potenciais riscos e benefícios. Pacientes ambulatoriais devem ser aconselhados a evitar atividades perigosas que exijam alerta mental e coordenação motora até a finalização do tratamento ${ }^{25}$.

Possui uma interação moderada com a soja, esses grãos podem estimular o crescimento do câncer de mama, interferindo, portanto, na ação do medicamento. Devido ao seu efeito estrogênico, as isoflavonas (presentes na soja) podem estimular o crescimento do tumor de mama antagonizando a ação do tamoxifeno, portanto os profissionais farmacêuticos devem orientar os nutricionistas e os médicos que não devem prescreverem alimentos à base de soja a esses pacientes durante o tratamento ${ }^{25}$.

Há interação com pacientes com história de trombose venosa profunda ou embolia pulmonar, pois há evidências de aumento dos eventos tromboembólicos. Portanto deve ser sempre avaliado seus parâmetros de coagulação 25 .

\section{Ciclofosfamida:}

A ciclofosfamida é um agente alquilante utilizado para o tratamento de neoplasias de mama, leucemias, tumores cerebrais, dentre outros, mieloma múltiplos, lúpus eritrematoso, esclerose sistêmica e artrite reumatoide ${ }^{26}$. Por ser um agente alquilante inativo é metabolizado no fígado, pelo sistema do citocromo P-450, que dará origem a vários metabólitos (após várias oxidações) como a fosforamida mostarda, este que exerce o efeito alquilante na cadeia de DNA ${ }^{27}$. 
Algumas reações adversas relacionadas ao uso deste medicamento são: mielosupressão, esterilidade, amenorreia, bradicardia, falência cardíaca, cistite, náuseas e vômitos. As reações de hipersensibilidade incluem urticária, angioedema, febre, calafrios, congestão nasal e ocular ${ }^{26}$.

A ciclofosfamida possui 347 interações medicamentosas, segundo Drugs.com, mas apenas 54 se apresenta como grave, exemplo fármacos utilizados no tratamento para HIV (tenofovir, emtricitabina). O uso do tenofovir tem sido associado com nefrotoxicidade relacionada a dose, portanto o farmacêutico deve juntamente com outros profissionais qual a melhor dose para o paciente, esse profissional também deve avaliar os testes de função renal frequentemente nos pacientes, e conversar com os pacientes e explicar que caso eles apresentem dor muscular, fraqueza muscular, dor óssea, devem relatar ao profissional médico ${ }^{28}$.

Possui 6 interações com doenças sendo elas, mielosupressão (podendo levar a infecções graves, devendo fazer avalição constante do hemograma do paciente), obstrução do trato urinário (pode causar cistite hemorrágica e hematúria, portanto deve verificar regularmente o sedimento urinário), pessoas que possuem comprometimento pulmonar (pode causar fibrose pulmonar) e disfunção renal (aumenta a toxicidade da ciclofosfamida), disfunção hepática (pode alterar a atividade terapêutica e a ativação metabólica do medicamento) e doença cardíaca (o medicamento pode causar insuficiência cardíaca congestiva e miocardite), mas somente as duas primeiras são consideradas interações graves ${ }^{28}$.

\section{Principal medicamento prescrito para o tratamento do câncer de próstata}

\section{Bicalutamida:}

A bicalutamida é um antiandrogênio não esteroide, sendo muito utilizada no tratamento da neoplasia da próstata. Ligase aos receptores androgênicos sem ativação da expressão genética, inibindo o estímulo androgênico e provocando uma regressão dos tumores da próstata ${ }^{29}$.

As reações adversas mais frequentes são: dor pélvica, astenia, constipação, diarreia e hematúria, edema periférico, sudorese, há relato de desenvolvimento de doença pulmonar intersticial após o início do tratamento e relato de toxicidade pulmonar $^{30}$.

Esse antiandrogênio possui 387 interações medicamentosas, sendo 61 consideradas graves, segundo Drugs.com, possui interação grave com amiodarona. A co-administração desses dois agentes pode aumentar o ritmo cardíaco, portanto deve monitorar os ritmos cardíacos frequentemente, o farmacêutico pode atuar monitorando e avaliando esses ritmos e também aconselhando os pacientes a relatarem quaisquer sintomas, principalmente se tiverem tonturas, desmaio, palpitações e falta de $a r^{31}$.

A terapia com esse medicamento deve ser administrada com precauções em pacientes com insuficiência hepática, pois há uma interação moderada entre o medicamento e a doença, este medicamento é metabolizado no fígado, podendo alterar o metabolismo da droga. Há relatos pós-comercialização de morte ou hospitalização devido a lesão hepática grave (insuficiência hepática) associada à bicalutamida, portanto, é recomendável cautela em pacientes com insuficiência hepática, eles devem ser orientados a relatar quaisquer sinais e sintomas de disfunção hepática, como icterícia e urina escura, recomenda-se monitorar clinicamente a função hepática ${ }^{31}$.

\section{Principal medicamento prescrito para o tratamento de leucemia mieloide crônica}

Imatinibe:

O mesilato de imatinibe é indicado para o tratamento de pacientes com leucemia mielóide crônica (LMC) em crise blástica, fase acelerada, ou em fase crônica da doença. Esse antineoplásico age competindo com o sítio da ATP da tirosinoquinase, restaurando seu mecanismo de morte celular, induzindo a remissão hematológica e citogenética na $\mathrm{LMC}^{32}$.

Os efeitos adversos, do imatinibe, se caracterizam como náuseas e vômitos, diarreias, cãibras musculares, dor muscular esquelética, hemorragia, erupções cutâneas, retenção de fluidos, dores de cabeça e edema ${ }^{33}$.

Esse medicamento possui 927 interações medicamentos, dentre elas 112 são consideradas graves como deflazacorte. O imatinibe pode aumentar significativamente os níveis sanguíneos do ativo do deflazacorte, podendo causar inchaço, fraqueza muscular, desequilíbrio eletrolítico e irregularidades menstruais, portanto o farmacêutico deve orientar o paciente a relatar quaisquer desses sintomas ao médico, o farmacêutico deve orientar o médico que deve ser utilizado doses baixas do deflazacorte caso necessite utilizar essas duas drogas concomitantes. A equipe multidisciplinar deve analisar os riscos e benefícios da utilização dessas drogas concomitante, para isso deve avaliar o paciente quanto aos efeitos colaterais sistêmicos de corticosteroides ${ }^{34}$.

Esse medicamento possui interação alimentar moderada com a toranja, essa fruta pode aumentar os níveis sanguíneos do imatinibe, portanto pacientes que consomem regularmente essa fruta, os pacientes devem ser monitorados quanto aos efeitos adversos e suas concentrações plasmáticas devem 
ser frequentemente analisados, o farmacêutico deve orientar tanto ao paciente, quanto ao nutricionista e o médico quanto ao risco que essa fruta pode causar quando se utiliza esse antineoplásico ${ }^{34}$.

Esse medicamente possui ainda 7 interações com doenças (todas elas classificadas como moderadas): doença cardiovascular (problemas cardíacos foram relatados durante tratamento com essa droga), retenção de fluidos (esse medicamento pode ocasionar retenção de líquidos), supressão de medula óssea (pode causar trombocitopenia e neutropenia), problemas gastrointestinais (esse medicamento pode causar irritabilidade gastrointestinal, nesse caso o farmacêutico deve orientar que o medicamento não pode ser tomado em jejum e tomar somente com água), insuficiência hepática (medicamento hepatotóxico) e renal (há uma maior exposição do medicamento) e hipotireoidismo (casos de hipotireoidismo foram relatados em pacientes com tireoidectomia) ${ }^{34}$.

\section{Principais medicamentos prescritos para o tratamento do câncer de pulmão}

\section{Erlotinibe:}

O erlotinibe é um antineoplásico utilizado principalmente para o tratamento de carcinoma pulmonar de células não pequenas (CPCNP), mas também é utilizado para o tratar o câncer colorretal e para os tumores de cabeça e pescoço, consiste num potente inibidor da tirosina-quinase seletivo do receptor para o fator de crescimento epidérmico (EGFR). Age inibindo competitivamente a ligação do ATP da tirosinoquinase ao receptor intracelular, impedindo assim a transmissão do sinal ao núcleo da célula ${ }^{35}$.

Os efeitos adversos mais comuns são diarreia, erupções cutâneas, mas os pacientes também podem apresentar vômitos, irritações nos olhos, febre, dor de cabeça, hemorragias, coceira, acne ou perda de cabelo, os efeitos menos frequentes são insuficiência renal, neutropenia e trombocitopenia ${ }^{36}$.

Segundo Drugs.com, esse medicamento possui 239 interações medicamentosas, mas apenas 16 delas são graves como por exemplo o omeprazol. A administração dessas duas drogas deve ser evitada, pois o omeprazol pode diminuir a biodisponibilidade oral do erlotinibe e reduzir suas concentrações plasmáticas. Portanto o profissional farmacêutico pode sugerir ao médico a substituição desse protetor gástrico por ranitidina por exemplo, caso o paciente não possa ficar sem um medicamento da classe dos protetores gástricos. $O$ farmacêutico também tem a finalidade de orientar o paciente que deve utilizar o erlotinibe por pelo menos 2 horas antes ou 10 horas após a administração da administração da ranitidina ${ }^{37}$.
Esse medicamento também possui interação moderada com a fruta toranja (esta pode aumentar os níveis do erlotinibe). Portanto pacientes que utilizam esse medicamento devem evitar o consumo da toranja, caso a equipe multidisciplinar ache necessário o consumo dessa fruta. Os alimentos de forma geral aumentam a absorção e a biodisponibilidade do erlotinibe, portanto este deve ser administrado por pelo menos uma hora antes ou 2 horas após a ingestão de alimentos ${ }^{37}$.

O medicamento apresenta cinco interações graves com as seguintes doenças: dermatites induzidas por drogas (este medicamento pode causar reações cutâneas, o farmacêutico deve orientar o paciente que o mesmo deve utilizar protetor solar para evitar toxicidades dermatológicas), distúrbios gastrointestinais (erlotinibe pode causar perfuração gastrointestinal), distúrbios oculares, pulmonares (pode causar doença pulmonar intersticial), insuficiência hepática (causa hepatotoxicidade) e renal (toxicidade renal) ${ }^{37}$.

\section{Principal medicamento prescrito para o tratamento do câncer cerebral}

\section{Temozolamida:}

A temozolamida é um medicamento antineoplásico, utilizado para o tratamento de neoplasias cerebrais e melanoma maligno metastático em estágio avançado. Seu mecanismo de ação é atuar no DNA da célula neoplásica, a fim de evitar a duplicação celular e interferindo, portanto, no crescimento do câncer ${ }^{38}$.

Os principais efeitos adversos causados pela temozolamida são: constipação, náusea, vômito, cefaleia, convulsão, fadiga, alopecia, perda de peso, convulsões, neutropenia ou linfopenia e trombocitopenia ${ }^{39}$.

Segundo Drugs.com esse medicamento possui 193 interações medicamentosas, sendo 36 delas consideradas graves, como a clozapina. A coadministração desse medicamento com a clozapina pode causar neutropenia podendo aumentar o risco e/ou gravidade da toxicidade hematológica. Portanto o farmacêutico pode atuar nesse caso sugerindo a troca ou suspensão da clozapina para o médico, ou monitorando o hemograma do paciente, avaliando principalmente os níveis de leucócitos no sangue do paciente ${ }^{40}$.

Possui interações com urticária (temozolamida é contraindicada em pacientes com histórico de alergias e urticárias), sendo essa de forma grave de interação, insuficiência hepática e renal e mielosupressão (pode causar anemia aplástica) ${ }^{40}$. 
Principais medicamentos prescritos para o tratamento de diversos tipos de câncer

\section{Doxorrubicina:}

A doxorrubicina é um quimioterápico pertencente ao grupo dasantraciclinas (antibióticos citostáticos). Esse medicamento é utilizado para o tratamento dos seguintes tumores: mama, pulmão, bexiga, tireoide e também carcinoma ovariano, sarcomas ósseos e dos tecidos moles, linfomas de Hodgkin e não-Hodgkin e leucemia linfoblástica aguda e leucemia mieloblástica aguda ${ }^{41}$. Atua como inibidor das enzimas topoisomerase I (topo I) e topoisomerase II (topo II) ${ }^{42}$.

O uso dessa droga é limitado devido à alta cardiotoxicidade, pode provocar também hepatotoxicidade e nefrotoxicidade. Esse medicamento também pode causar fadiga extrema, anorexia e desconforto muscular, vômitos, diarreia, náuseas e sangramentos ${ }^{42}$.

A doxorrubicina, possui 548 interações medicamentosas, mas apenas 92 delas são consideradas graves, sendo haloperidol um exemplo dessa interação. $\mathrm{O}$ uso do haloperidol concomitante com esse anti-neoplásico pode aumentar o risco de arritmias cardíacas. O farmacêutico deve orientar os pacientes a procurarem o médico se apresentarem sintomas como tonturas, desmaios, palpitações, falta de ar e arritmias cardíacas, esses profissionais também devem conversar com o médico para avaliar os riscos e benefícios da administração dessas duas drogas concomitantes ${ }^{43}$.

O uso desse medicamento pode ser contraindicado em pacientes com doenças infecciosas conhecidas, pois pode induzir a mielossupressão devido ao efeito citotóxico da droga, o farmacêutico neste caso tem a função de orientar os pacientes para relatarem o médico caso apresentem quaisquer sinais e sintomas de infecção como febre, dor de garganta e/ ou infecções, também deve auxiliar no monitoramento da função hematopoiética ${ }^{43}$.

\section{Paclitaxel:}

O paclitaxel é um quimioterápico utilizado para o tratamento tumor de ovário, mama, pulmão, sarcoma de Kaposi, também pode ser usado para tratar tumores gastroesofágicos, endometriais, cervicais, próstata, cabeça e pescoço, linfomas e leucemias. São agentes específicos do ciclo celular que se liga ao microtúbulos, estabilizando-os, inibindo a fase mitótica das células cancerígenas, levando a morte celular por apoptose $\mathrm{e}^{44 ; 45}$.

O paclitaxel pode causar dispneia, dor abdominal, hiperemia, tremores de frio, dor lombar, mal-estar geral, sensação de calor, náuseas, vômitos e alopecia ${ }^{46}$.

Esse antioneplásico possui 406 interações medicamen- tosas, sendo 50 delas graves, como a clozapina. A clozapina pode diminuir a contagem de leucócitos (neutropenia) e administra-la juntamente com o paclitaxel pode aumentar o risco de disfunção medular, podendo o paciente a desenvolver infecções graves e/ou fatais. Portanto o profissional farmacêutico pode auxiliar no monitoramento da contagem de leucócitos, e explicar ao paciente os riscos que o uso desses dois medicamentos pode causar, e pedir para que o paciente relate caso apresente reações adversas, como febre, calafrios, diarreia, dor de garganta, dores musculares, falta de ar, perda de peso, feridas, vermelhidão, entre outros ${ }^{47}$.

A fruta toranja possui interação grave com esse medicamento, podendo aumentar os níveis sanguíneos e os efeitos do paclitaxel. Recomenda-se suspender o consumo dessa fruta, caso seja necessário a utilização dessa fruta na dieta do paciente, o farmacêutico deve auxiliar na monitoração do paciente, observando casos de toxicidade pelo medicamento, incluindo diarreia, mielossupressão e neuropatia periférica, deve auxiliar também no ajuste da dose do medicamento ${ }^{47}$.

Esse medicamento possui interação grave com pacientes que possuem infecções (devido ao efeito citotóxico da droga pode ocorrer a mielossupressão), pacientes que possuem ou que apresentam predisposição à distúrbios de condução (há relatos de anormalidades severas de condução durante a terapia com paclitaxel), pacientes com disfunção hepática (medicamento metabolizado no fígado, portanto risco de hepatotoxicidade), com mielossupressão (medicamento induz a mielossupressão) e pacientes com neuropatia periférica (depende da dose do medicamento) e possui ainda interação moderada com pacientes que possuem predisposição a ter anafilaxia (medicamento pode causar reações graves de hipersensibilidade ${ }^{47}$.

\section{Principal medicamento prescrito para o tratamento do câncer renal}

\section{Pazopanibe:}

Esse quimioterápico é um potente inibidor da tirosinaquinase, dirigidos aos receptores do fator de crescimento endotelial vascular 1, 2 e 2 (VEGFR-1, VEGFR-2 e VEGFR-3), do fator de crescimento derivado de plaquetas a e $\beta$ (PDGFR- $a$ e PDGFR- $\beta$ ) e receptor de células troncos (c-KIT71). Essa droga mostrou-se ativo em monoterapia para pacientes com câncer avançado ou metastático de células renais ${ }^{48}$.

Os efeitos adversos mais comuns desse medicamento, foram diarreia, exantema e náuseas pode apresentar também disfunção hepática ${ }^{49}$.

Segundo Drugs.com, esse medicamento possui 698 interações medicamentosas, sendo 179 delas de modo 
grave, sendo omeprazol exemplo dessas interações. A coadministração desse medicamento com o inibidor da bomba de prótons pode diminuir significativamente a biodisponibilidade do pazopanibe, reduzindo assim suas concentrações plasmáticas, portanto deve se evitar a administração concomitante dessas duas drogas, mas caso o paciente precise continuar tomando esse protetor gástrico, deve-se tomar o pazopanibe por pelo menos 2 hora antes ou 10 horas após a administração do omeprazol ${ }^{50}$.

Esse antineoplásico também possui interação com a toranja (aumenta a concentração plasmática do pazopanibe), pois pode levar a um aumento do risco de efeitos secundários graves, tais como danos no fígado, ritmo cardíaco irregular, hemorragia, pressão alta, ataque cardíaco e Acidente Vascular Cerebral (AVC), portanto deve-se evitar o consumo dessa fruta na dieta do paciente durante o tratamento. Os alimentos aumentam a biodisponibilidade do medicamento (consumo de alimentos ricos em gorduras ou com baixo teor de gordura resulta num aumento de até duas vezes o pico plasmático da droga), portanto deve-se administrar o medicamento por pelo menos 1 hora antes ou 2 horas após a ingestão de alimentos ${ }^{50}$.

Possui também 11 interações com doenças, sendo as interações graves, pacientes que apresentem sangramentos (medicamento pode aumentar o risco de sangramento), disfunção hepática (pode causar hepatotoxicidade grave ou fatal), pacientes com ou com predisposição a obstrução gastrointestinal (pode causar perfuração gastrointestinal e fístulas) e interações moderadas, pacientes que possuem doença cardiovascular, hipotireoidismo (droga pode causar hipotireoidismo), disfunção pulmonar (há relato de doença pulmonar intersticial e/ou pneumonite), com Síndrome do QT longo (pode aumentar o risco de arritmias cardíacas), hipertensão (pode aumentar a pressão arterial), Síndrome da Encefalopatia Reversível Posterior (durante o tratamento com esse medicamento, houve relato dessa doença), proteinúria (aumento da gravidade e da incidência) e distúrbios tromboembólicos (relatos de eventos tromboembólicos venosos e arteriais) ${ }^{50}$.

Portanto, a equipe multidisciplinar terá que fazer uma avaliação do quadro do paciente, verificar a história familiar da mesma, se possui alguma doença, se toma algum medicamento, analisar a dose ideal para a paciente, sua dieta, e principalmente o custo benefício do tratamento. Em caso de o paciente utilizar algum medicamento que possui interação medicamentosa grave com algum desses medicamentos antineoplásicos, verificar se há a possibilidade de substituição do medicamento em uso até a finalização do tratamento, ou se poderá substituir o medicamento oncológico por outro.

\section{Conclusão}

O profissional farmacêutico deve prestar uma excelente atenção farmacêutica em paciente oncológicos, para garantir que os mesmos tenham uma melhor qualidade de vida e sigam o tratamento corretamente e sem muito sofrimento.

A principal função do farmacêutico que atua na área da oncologia é colaborar com a equipe multidisciplinar, discutindo sobre os procedimentos oncológicos mais aprimorados para cada paciente e evitando interações medicamentosas graves. E também, juntamente com essa equipe, deve escolher a melhor opção de tratamento medicamentoso para cada paciente, levando em consideração ao estilo de vida de cada doente.

O farmacêutico também tem uma grande responsabilidade em orientar os pacientes quanto ao tratamento medicamentoso ou não, explicando que caso o mesmo opte por não se tratar por meio desses medicamentos, que eles poderão ter sérias consequências, como por exemplo a morte. O farmacêutico tem a função de explicar que as drogas antineoplásicas tem efeitos colaterais graves, mas que o risco de benefício é melhor do que a opção de não seguir com o tratamento.

O farmacêutico também deve orientar o paciente que o mesmo sempre relate os sinais e sintomas que o mesmo está tendo, pois assim o farmacêutico poderá juntamente com a equipe discutir novas opções de tratamento, caso esses sintomas seja um efeito colateral do medicamento.

Portanto, o farmacêutico atuante da área de oncologia, deve ser um profissional comprometido em cuidar de paciente debilitados, fragilizados fisicamente e psicologicamente, também deve saber orientar tanto os pacientes quanto seus familiares, utilizando palavras sensatas e de conforto. Assim sendo é um profissional que não pode faltar na equipe multidisciplinar da oncologia.

\section{Referências}

1. Leão AM, Neves E, Dias JP, Santos PK. Atenção farmacêutica no tratamento oncológico em uma instituição pública de montes claros-mg. Rev Bras Farm Hosp Serv Saúde. 2012;3(1):11-14.

2. Oliveira e Souza JAA, Cordeiro BC. Atenção farmacêutica às pacientes oncológicas de um hospital de grande porte do Rio de Janeiro. Rev Bras Farm Hosp Serv Saúde. 2012;3(2):6-9.

3. Provin MP, Campos AP, Nielson SEO, Amaral RG. Atenção farmacêutica em Goiânia: inserção do farmacêutico na estratégia saúde da família. Rev Saúde Soc. 2010;19(3):717-723.

4. Brazil LOS. O câncer e as alterações no estado nutricional do paciente: importância da atenção farmacêutica 
[Trabalho de Conclusão de Curso]. Araraquara-SP: Universidade Estadual - UNIP; 2011.

5. Bovo F, Wlsniewski P, Morskei MLM. Atenção farmacêutica: papel do farmacêutico na promoção da saúde. Rev Biosaúde. 2009;11 (1):43-56.

6. Meneses ALL, Sá MLB. Atenção farmacêutica ao idoso: fundamentos e propostas. Rev Geria Geront. 2010;4(3):154-161.

7. Silva RM, Soares RSA, Lana LD, Birrer JA, Mostardeiro SCTS. Orientações pré-operatórias recebidas por pacientes submetidos à cirurgia oncológica. Rev Cient Sena Aires. 2017;6(2):116-25.

8. Oliboni LS, Camargo AL. Validação da prescrição oncológica: o papel do farmacêutico na prevenção de erros de medicação. Rev do Hosp Clín Facul Med. 2009;29(2):147-152.

9. Pinho MS, Abreu PA, Nogueira TA. Atenção farmacêutica a pacientes oncológicos: uma revisão integrativa da literatura. Rev Bras Farm Hosp Serv Saúde. 2016;7(1):33-39.

10. Santos H, Batista G, Mota G, Martins M, Nunes S. Atribuições do farmacêutico em unidade de assistência de alta complexidade em oncologia. Rev Infarma. 2013;25(1):40-44.

11. Rodrigues JSM, Ferreira NMLA. Caracterização do perfil epidemiológico do câncer em uma cidade do interior paulista: conhecer para intervir. Rev Bras Canc.2010;56(4):431-441.

12. Instituto Nacional do Câncer. Estimativa 2018 - Incidência de Câncer no Brasil. INCA, Rio de Janeiro-RJ. 2018.

13. LIhara $\mathrm{H}$, Ishihara $M$, Matsuura $K$, Kurahashi S, Takahashi T, Kawaguchi $Y$, et al. Pharmacists contribute to the improved efficiency of medical practices in the outpatient cancer chemotherapy clinic. J Eval Clin Pract. 2012;18(4):753-60.

14. Eugênio LSG, Pinheiro OL. Paciente oncológico em tratamento medicamentoso: subsídios para implantação de um programa de atenção farmacêutica. Rev Temas Saúde. 2018;18(2):216-237.

15. Silva AG, Azevedo C, Mata LRF, Vasques Cl. Adesão de pacientes ao tratamento com antineoplásicos orais: fatores influentes. Rev Baiana Enf. 2017;31(1):1-12.

16. Oliveira AT, Queiroz APA. Perfil de uso da terapia antineoplásica oral: a importância da orientação farmacêutica. Rev Bras Farm Hosp Serv Saúde. 2012;3(4):24-29.

17. Frias TFP, Costa CMA, Sampaio CEP. O impacto da visita pré-operatória de enfermagem no nível de ansiedade de pacientes cirúrgicos. Rev. Min Enferm.
2010;14(3):345-352.

18. Lourenço AV. Women cancer prevention and pharmaceutical contribution. Braz J Pharm Sci. 2010;46(1):45-52.

19. Martins TL, Pinto AR, Pires JMD, Silva MJS, Calil-Elias DHN. Reação adversa induzida por capecitabina: a importância da farmacovigilância. Rev Bras Farm Hosp Serv Saúde. 2013;4(3):24-26.

20. Lunardi D, Zart D, Fasolin T, Gonçalves CBT. Atenção farmacêutica para pacientes em uso de capecitabina. Rev Bras Farm. 2009;90(3):250-257.

21. Gifoni MAC. Mucosite E. Alterações de permeabilidade intestinal em pacientes portadores de câncer colorretal metastático tratados com 5 -Fluorouracil (5-Fu) e Irinotecano (Cpt-11) [Tese de Doutorado]. Fortaleza, CE: Fundação Antônio Prudente; 2012.

22. Drugs.com [Internet].; 2019 [acesso em 04 de Março de 2019]. Disponível em: https:/www.drugs.com/druginteractions/capecitabine.html.

23. Bönmann TJ, Lissarassa YPS. Principais efeitos colaterais e alterações endometriais relacionadas ao uso do tamoxifeno em tratamento de câncer de mama. Rev. Saúde Int. 2016;9(2):25-28.

24. Marianito SIG. O papel da farmacogenómica na terapêutica com tamoxifeno [Disertação de Mestrado]. Faro- Portugal:, Universidade Do Algarve; 2012.

25. Drugs.com [Internet]; 2019 [acesso em 04 de Março de 2019]. Disponível em: https://www.drugs. com/drug-interactions/escitalopram-with-tamoxifen-1013-0-2145-0.html?professional=1.

26. Teles ACVSC. "Estado de arte? no tratamento do carcinoma da próstata [Dissertação de Mestrado]. Coimbra-Portugual: Universidade de Coimbra; 2015.

27. Farias RC, Souza PM, Mota LMH, Ferreira F. Estabilidade de ciclofosfamida e suas preparações parenterais: uma revisão [Trabalho de Conclusao de Curso]. Brasília-DF: Universidade de Brasília - UNB.; 2015.

28. Drugs.com [Internet]; 2019 [acesso em 04 de Março de 2019]. Disponível em: https://www.drugs. com/drug-interactions/cyclophosphamide-withefavirenz-emtricitabine-tenofovir-761-0-964-0. html?professional=1.

29. Teles KA, Medeiros-Souza P, Lima FAC, Araújo BG, Lima RAC. Rotina de administração de ciclofosfamida em doenças autoimunes reumáticas: uma revisão. Rev Bras Reumatol 2017;57(6):596-604.

30. Mancero GM, Picon X, Di Tullio F, Ernst G, Dezanzo P, Salvado $A$, et al. Neumonía Intersticial Inducida Por Bloqueo Androgénico Máximo Como Tratamiento 
De Cáncer De Próstata Avanzado. Rev Méd Chile. 2016;144(10):1356-1359.

31. Drugs.com [Internet]; 2019 [acesso em 04 de Março de 2019]. Disponível em: www.drugs.com/drug-interactions/amiodarone-with-bicalutamide-167-0-380-0. html?professional=1.

32. Bollmann PW, Giglio A. Leucemia mieloide crônica: passado, presente, futuro. Rev Einstein. 2011;9(2):236-43.

33. Cruz PS, Santos MLSC, Santos WA, Trovatti P, Fuly PSC. Qualidade de vida dos pacientes com leucemia mieloide crônica em uso de imatinibe. Rev. de Enferm UFPE On-Line. 2017;11(6):2423-31.

34. Drugs.com [Internet]; 2019 [acesso em 04 de Março de 2019]. Disponível em: https://www.drugs.com/druginteractions/deflazacort-with-imatinib-3785-0-1321-0. html?professional=1.

35. Göller FF, Moreira AC. Terapia-alvo em oncologia: erlotinib. Rev Infarma. 2010;22(9/10):22-24.

36. Gontijo SMD. Avaliação da citotoxicidade do composto de inclusão de erlotinibe em hidroxipropil-beta-ciclodextrina e da hipertermia associada à cisplatina [Tese de Doutorado]. Belo Horizonte- MG: Universidade Federal de Minas Gerais -UFMG; 2015.

37. Drugs.com [Internet]. 2019 [acesso em 04 de Março de 2019]. Disponível em: https://www.drugs.com/drug-interactions/erlotinib-with-omeprazole-1007-0-1750-0. html?professional=1.

38. Vedrame L, Raffin RP, Fagan SG, Silva IZ. Simulação de primeiros princípios de temozolamida interagindo com nanoestruturas. Rev Disc Scientia. Série: Naturais e Tecnológicas. 2015;16(2):231-243.

39. Schiavo M. Guia para dispensação de quimioterápicos via oral [Trabalho de Conclusão de Curso]. Ijuí-RS: Universidade Regional do Noroeste do Estado do Rio Grande do Sul - Unijuí; 2017.

40. Drugs.com [Internet]; 2019 [acesso em 04 de Março de 2019]. Disponível em: https://www.drugs. com/drug-interactions/clozapine-with-temozolomide-709-0-2154-0.html?professional=1.

41. Martins WA, Souza VB, Rocha LFC, Villacorta Júnior $H$. Cardiomiopatia por doxorrubicina em pacientes com câncer de mama- a propósito de dois casos. Rev Bras Cardiol. 2010;24(3):196-198.

42. Moura LE, Viegas AA, Nasciutti PR, Carvalho ROA, Moura VMBD. Cardiotoxicidade induzida pela doxorrubicina: patogenia, diagnóstico e terapias antioxidantes. Rev Enc Biosfera. 2015;11(22):711-736.

43. Drugs.com [Internet]; 2019 [acesso em 04 de
Março de 2019]. Disponível em: https://www.drugs. com/drug-interactions/doxorubicin-with-haloperidol-938-0-1230-0.html?professional=1.

44. Weaver BA. How taxol/paclitaxel kills cancer cells. Rev Mol Biol Cell. 2014;25(18):2677-2681.

45. Miele E, Spinelli GP, Tomao F, Tomao S. Albumin-bound formulation of paclitaxel (abraxane ${ }^{\circledR}$ abi-007) in the treatment of breast cancer. Int Jour Nanomedicine. 2009;4: 99-105.

46. Aguiar TOD, Capucho HC, Varallo FR, Mastroianni PC. Notificações espontâneas de eventos adversos a medicamentos hospitalares: estudo piloto. Rev Ciênc Ex. 2015;11(1):46-61.

47. Drugs.com [Internet; 2019 [acesso em 04 de Março de 2019]. Disponível em: https://www.drugs.com/druginteractions/clozapine-with-paclitaxel-709-0-1778-0. html?professional=1.

48. Leite CAVG, Costa JVG, Callado RB, Torres JNL, Lima Júnior RC, Ribeiro RA. Receptores tirosina-quinase: implicações terapêuticas no câncer. Rev Bras Onc Clín. 2012;8(29):130-142.

49. Miranda CSC. Alvos terapêuticos no cancro da mama novas perspectivas [Monografia de Estágio]. Coimbra - Portugual: Universidade de Coimbra; 2015.

50. Drugs.com [Internet]; 2019 [acesso em 04 de Março de 2019]. Disponível em: https://www.drugs. com/drug-interactions/esomeprazole-with-pazopanib-1015-0-3180-0.html?professional=1. 